\title{
EXPERIMENTACIÓN CON ESPECIES LEÑOSAS DE LA PUNA ME- RIDIONAL DE ARGENTINA. APORTES A LOS ESTUDIOS ANTRACOLÓGICOS.
}

\author{
EXPERIMENTATION ON WOODY PLANT SPECIES FROM \\ SOUTHERN ARGENTINEAN PUNA. CONTRIBUTIONS TO \\ ANTHRACOLOGICAL STUDIES
}

\author{
María Gabriela Aguirre ${ }^{1}$ \\ María Fernanda Rodríguez ${ }^{2}$ \\ 1.Facultad de Ciencias Naturales e IML. UNT. Miguel Lillo 205. CP 4000. Argentina. \\ E-mail: mgabaguirre@hotmail.com \\ 2Instituto Nacional de Antropología y Pensamiento Latinoamericano, 3 de Febrero 1378, C1426BJN, Ciudad \\ Autónoma de Buenos Aires. Argentina. E-mail: mfrodriguez18@gmail.com
}

Presentado el: 21/02/2013/ - Aceptado el: 28/08/2013

\begin{abstract}
Resumen
En este trabajo se investigó experimentalmente la reacción de ocho especies leñosas ante la acción del fuego. Para esto se planteó un diseño de experimentación de quemas de leña que se llevó a cabo en el área de estudio: Antofagasta de la Sierra, Catamarca. Las especies leñosas seleccionadas para la experimentación fueron aquellas que se usan actualmente como leña en el área de estudio, de acuerdo con las entrevistas etnobotánicas realizadas y los taxones identificados en fogones arqueológicos del área. Los ensayos se realizaron en dos fogones: en cubeta y en piso. Se discuten los resultados obtenidos confrontándolos con el registro arqueológico del área. Este trabajo constituye el punto de partida hacia un nuevo modo de analizar el registro arqueobotánico en relación con el uso de los recursos leñosos en el pasado.
\end{abstract}

Palabras claves: Antracología, Especies leñosas, Estudios experimentales, Puna meridional argentina

\footnotetext{
Abstract

The main objective of this research was to describe the reaction of each woody plant species in front of the fire. So, we proposed an experimental design of firewood burning that we carried out in the study area: Antofagasta de la Sierra, Catamarca. This work constitutes the starting point toward a new way to analyze the archaeobotanical record in relation to the use of woody resources
} 
in the past. The selected woody species were those used at present day as firewood in the study area, considering the ethnobotanical interviews, and the identified taxa from archaeological camp fires in the area. The results are discussed and, then, confronting with the archaeobotanical record of the area.

Key words: Anthracology, Experimental studies, Woody plant species, Southern argentinean puna.

\section{Introducción}

El fuego y su uso a lo largo de la historia de la humanidad se convirtieron en temas de interés para diferentes disciplinas, entre ellas la arqueología. La conjunción de los estudios arqueológicos y de los métodos y técnicas de la xilología han permitido la identificación anatómico-taxonómica de restos leñosos y de carbón recuperados en diversos contextos arqueológicos. Al mismo tiempo, los aspectos relacionados con la recolección, selección y transformación del material leñoso, se han abordado a través de la consulta de fuentes etnográficas en general y etnobotánicas en particular, lo cual contribuye a las interpretaciones arqueológicas ya que permite distinguir a escala temporal actual o subactual, distintas formas de relación entre los grupos humanos y la vegetación (Zapata Peña et al. 2003, González Urquijo et al. 2005; Picornell Gelabert 2009).

Otro aspecto relevante en el estudio de los recursos combustibles se relaciona con la evaluación de las propiedades cuantitativas y cualitativas de los mismos. El valor calorífico, la densidad de la madera, el contenido de humedad, las cenizas, la velocidad de secado y la composición química forman parte de las propiedades cuantitativas, entre las cuales es posible establecer relaciones, por ejemplo, cuanto más húmeda es la madera menos eficiente será como combustible ya que el valor calorífico neto se reduce, variando a su vez el contenido de humedad. Esta última depende de las dimensiones de las ramas y de las estaciones del año, motivo por el cual, el contenido de agua no debe ser considerado como un valor intrínseco de una especie combustible. Se considera que el valor calorífico, la densidad de la madera y las cenizas son las propiedades más adecuadas para determinar la capacidad de una madera como combustible (Nirmal Kumar et al. 2011). Por otra parte, las propiedades cualitativas se refieren a las categorizaciones de las leñas realizadas por las comunidades que emplean este tipo de combustibles en actividades cotidianas.

Puede decirse entonces que el estudio de los recursos combustibles requiere de abordajes integrales que contemplen tanto los aspectos sociales implicados en la selección y uso de las leñas como la consideración de las características físicos-químicas de las mismas. Se suman los estudios experimentales que han demostrado ser sumamente útiles en los análisis antracológicos (March 1992, Allué et al. 2005, Théry-Parisot et al. 2010) y es en este campo en donde se inserta esta investigación.

Diversos estudios antracológicos se han desarrollado en la localidad de Antofagasta de la Sierra (Provincia de Catamarca). Los mismos se llevaron a cabo principalmente en las localidades arqueológicas de Quebrada Seca (Rodríguez 1998, 2000), Punta de la Peña (Rodríguez 2004), Peñas Chicas (Aguirre 2007), Quebrada Miriguaca (Aguirre 2012) y en el sector de Fondo de Cuenca (Joly 2008). Dichos estudios permitieron identificar las especies leñosas utilizadas como combustible por los grupos que habitaron el área, el radio de captación de las mismas y las asociaciones vegetales de las cuales proceden. 
El conocimiento generado suscitó una serie de interrogantes referidos a las características de los conjuntos de carbones analizados. Nos planteamos preguntas en relación con la composición taxonómica de los fogones: ¿Por qué recuperamos estos taxones y no otros? ¿Qué tipo de factores intervienen en la composición taxonómica de los fogones? y preguntas acerca de los momentos del manejo del fuego que estamos interpretando arqueológicamente: ¿analizamos todos los taxones quemados o sólo los últimos agregados al fuego? ¿Registramos el apagado natural o intencional del fogón?

Así, el objetivo general de esta investigación es describir la reacción de ciertas especies leñosas ante la acción del fuego. Al mismo tiempo, indagar los factores naturales y antrópicos que intervienen en la composición de un fogón arqueológico.

\section{Características ambientales del área de estudio}

La localidad de Antofagasta de la Sierra forma parte de la Puna Salada (Troll 1958), específicamente del sector meridional caracterizado por grandes salares y escasos cursos de agua (Cabrera 1976). Desde el punto de vista fitogeográfico, pertenece a la Provincia Puneña del Dominio Andino en donde la vegetación dominante es la estepa arbustiva (Cabrera 1976, Cabrera y Willink 1980). La cuenca de Antofagasta de la iblSierra comprende el sistema hídrico de los ríos Calalaste-Toconquis-Punilla/ Antofagasta y sus afluentes, dentro de los cuales se destacan los ríos Las Pitas y Miriguaca.

Los suelos son inmaduros, pobres en materia orgánica (Cabrera 1976, Martínez Carretero 1995) y el clima puede describirse como seco y frío, con lluvias escasas y estivales. Las comunidades vegetales varían en composición y abundancia de acuerdo con el

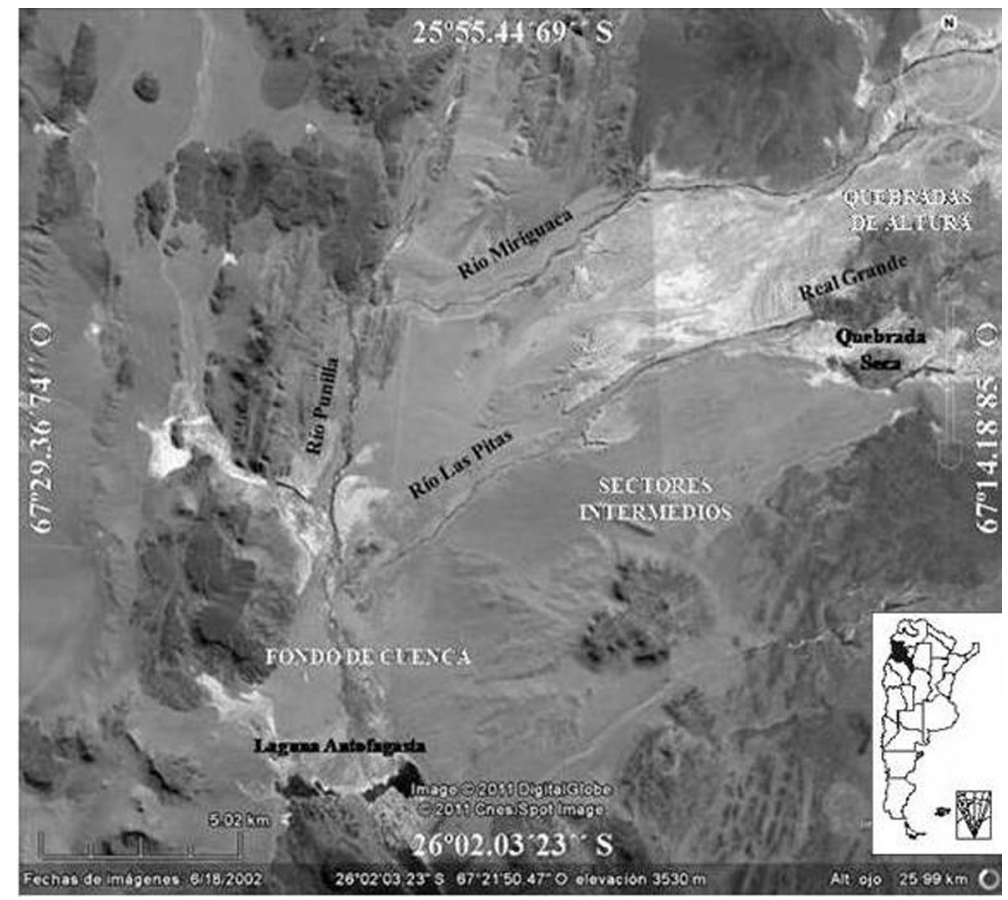

Figura 1 Localidad de Antofagasta de la Sierra (Provincia de Catamarca, Argentina). Se indican los principales ríos que integran la cuenca homónima y los tres sectores topográficos diferenciados 
gradiente altitudinal y con la distancia a cuerpos y flujos de agua; de este modo, es posible distinguir vegetación de tipo disperso (faldeos de Quebradas, Campos y Pampas) y agrupado (Vegas).

Para esta localidad se propone una sectorización que contempla tres espacios diferenciados: Fondo de Cuenca (3.400-3.550 msm), Sectores Intermedios (3.500-3.800 msm) y Quebradas de Altura (3.900-4.900 msm) (Olivera 1992) (Figura 1). En estos sectores pueden identificarse distintas unidades vegetacionales, tales como Vega, Tolar, Campo y Pajonal (Haber 1992). Cada una de ellas presenta distintos porcentajes de cobertura vegetal, así como también de composición florística.

\section{Experimentación y antracología}

En arqueología los experimentos tienen como finalidad descubrir, describir, explicar y predecir distintos aspectos relacionados con diferentes vestigios arqueológicos (Nami 1991). Coles (1979) señala que la arqueología experimental provee un camino para examinar supuestos arqueológicos sobre el comportamiento humano en el pasado. Por otra parte, esta disciplina en tanto rama de la arqueología, incluye la replicación, el análisis y/o la interpretación de materiales arqueológicos por medio de la experimentación científica (Ascher 1961).

Scarano et al. (1994) sintetizan las principales formas de investigaciones experimentales, la primera es el estudio observacional controlado: "que consiste en la búsqueda de variadas situaciones para determinar si un fenómeno se produce uniformemente de la misma manera, si el mismo varía, o bien si tiene lugar sólo en algunos casos pero no en otros" (Scarano et al. 1994:192). Por otra parte, en los experimentos de campo el sistema experimental es "conscientemente seleccionado, estable y controlable; además se puede describir precisamente y variar dentro de cierta gama uno o varios factores" (Scarano et al. 1994:193). Por último, en los experimentos en sentido estricto, "el experimentador puede manipular a voluntad, aunque sólo dentro de determinados límites, ciertos aspectos de una situación (factores o variables) de los que supone que constituyen las condiciones para la aparición de los fenómenos estudiados" (Scarano et al. 1994:193). March et al. (2012) en un estudio sobre las estructuras de combustión arqueológicas, señalan la importancia de haber desarrollado una metodología basada en una aproximación interdisciplinaria que ligó aspectos físicos, químicos, estudios de modelización y simulaciones experimentales; estas últimas ilustran la complejidad del proceso de formación de las estructuras asociadas al fuego y clarifican sobre el significado de cada elemento que compone a las mismas.

Otros aspectos relevantes en el estudio del uso del fuego y las estructuras de combustión, corresponden a la tafonomía y a los procesos de formación. El término tafonomía inicialmente propuesto por Efremov en 1940, aludía a la disciplina que estudia "las leyes de enterramiento", posteriormente el mismo término fue redefinido por otros autores (Behrensmeyer y Kidwell 1985) evolucionando su sentido de acuerdo con el modo utilizado en diferentes áreas de investigación (March et al. 2012). Así, en estudios antracológicos, es importante evaluar los distintos agentes tafonómicos que actúan sobre los carbones y fogones. En cuanto a los procesos de formación (natural o cultural) (Schiffer 1972, 1975) se reconoce que tanto la naturaleza como los seres humanos afectan y transforman al registro arqueológico. De ahí que un estudio antracológico, además de orientarse a identificar taxonómicamente las especies leñosas empleadas y reconocer sus áreas de procedencia, debe llevarse a cabo teniendo en cuenta aquellos eventos, actividades y procesos que afectan al registro arqueológico. 
La madera está constituida por diversos polímeros que conforman un complejo de células interconectadas con orientaciones específicas, siendo la celulosa el principal polímero (Byrne y Nagle 1997). La molécula de celulosa está formada por largas cadenas radicales de glucosa unidas entre sí por enlaces (Fahn 1974). El segundo polisacárido más importante en las maderas es la hemicelulosa, seguido por la lignina que comprende entre 18 y 35 \% del peso de las maderas secas; este compuesto otorga rigidez y dureza a las paredes celulares y las regiones intercelulares (Byrne y Nagle 1997).

De acuerdo con el origen biológico, las maderas pueden dividirse en blandas (derivadas de las coníferas) y duras (derivadas de las angiospermas); ambos tipos difieren en sus contenidos de lignina, celulosa y hemicelulosa (Braadbaart y Poole 2008). Cuando las maderas son expuestas al fuego sufren una descomposición térmica durante la cual la celulosa se transforma en carbón sólido (Byrne y Nagle 1997). La formación de carbón ocurre por calentamiento de la madera en un ambiente con suministro limitado de oxigeno (quema) o en un ambiente carente de oxigeno (carbonización). De este modo, las propiedades morfológicas, físicas y químicas del carbón dependerán de dos variables: fuente de calor (temperatura, tiempo de exposición) y propiedades de la madera (taxón, tamaño entre otros) (Braadbaart y Poole 2008). En el proceso de combustión pueden distinguirse diferentes etapas a medida que aumenta la temperatura:

-La primera etapa consiste en la deshidratación, la cual coincide con la ruptura de las moléculas de hemicelulosa; en esta etapa y durante la siguiente ocurre la pérdida de peso del material en un 35\% (Badal et al. 2003).

-La segunda etapa se conoce como torrefacción (Badal et al. 2003). A los $150^{\circ} \mathrm{C}$ ocurre la desorción del agua, mientras que entre los $150^{\circ}-240^{\circ} \mathrm{C}$ ocurre la división de la estructura de la molécula de celulosa y entre los $240^{\circ}-400^{\circ} \mathrm{C}$ se produce la depolimeración de la celulosa, rompiéndose los enlaces C-O y C-C (Byrne y Nagle 1997).

-La pirólisis o carbonización conduce a las brasas (Badal et al. 2003). La pirolisis no progresa a tasas de temperatura establecidas, por ejemplo, la ruptura de la lignina ocurre en esta etapa entre los $280^{\circ}-500^{\circ} \mathrm{C}$ (Byrne y Nagle 1997). Cuando la carbonización ocurre en ausencia de aire, la formación de gases como el $\mathrm{CO}$ y CO2, ocurre entre los $170^{\circ}$ y $275^{\circ} \mathrm{C}$, al superarse estas temperaturas, disminuye la producción de estos gases (Bueno Zarate 1987) y aumenta la producción de metano, hidrógeno y etano cuando las temperaturas alcanzan los $252^{\circ}$ a $352^{\circ} \mathrm{C}$ (Demirbas 2004).

-Por último, la cumburación o etapa de generación de cenizas (Badal et al. 2003), lleva a la descomposición completa de los materiales. El proceso de formación de cenizas es dependiente de la técnica de combustión empleada y de la especie leñosa que haya sido expuesta al calor, siendo variables los elementos formadores de ceniza como también su presencia en diferentes partes del cuerpo de un árbol (corteza, madera, ramas menores, brotes) (Werkelin et al. 2011).

El proceso de combustión y sus resultados fueron estudiados de manera experimental por diferentes autores. Prior y Alvin (1983) estudiaron las modificaciones anatómicas del leño de maderas de diferentes especies luego de la carbonización. Braadbaart et al. (2009) generaron carbones experimentales que fueron expuestos a condiciones de alcalinidad a fin de simular condiciones de suelos con ese tipo de $\mathrm{pH}$ debido a que estas condiciones 
intervienen en la preservación de los restos carbonosos. Por su parte, Mc Parland et al. (2010) analizaron el proceso de vitrificación bajo condiciones controladas con el objetivo de relacionarlo con carbones procedentes de contextos arqueológicos europeos.

Otras vías de investigación fueron las llevadas a cabo por Allué et al. (2005) quienes realizaron un estudio para analizar la cuantificación, la reducción de masa y las deformaciones anatómicas de la madera antes y después de la combustión. Un aspecto importante señalado por estos autores es la imposibilidad de reproducir con exactitud la forma de recolección de leña realizada por los cazadores recolectores del Paleolítico, siendo la misma dependiente de los individuos que realizan esta acción en cuanto a forma y cantidad. Por este motivo, es necesario comparar luego los resultados de las experimentaciones con datos etnográficos.

March (1992), a través de la realización de fogones experimentales, estudió la relación entre el tipo de madera utilizada y su rendimiento expresado en $\mathrm{kg} / \mathrm{h}$, además de tratar de comprender la relación entre la forma de los fogones y los carbones resultantes con el fin de relacionar estos datos experimentales con datos provenientes de contextos cazadoresrecolectores. De acuerdo con los resultados obtenidos, este autor considera que los mejores rendimientos se dan en fogones en cubeta más que en fogones planos y al mismo tiempo producen más carbón que los fogones planos. Por su parte, Théry-Parisota et al. (2010) señalan la complejidad del proceso de combustión sugiriendo que las condiciones experimentales son las que en realidad determinan los efectos en los residuos carbonosos. March et al. (2012) presentan un enfoque experimental para estudiar los procesos de formación y transformación de las estructuras de combustión arqueológicas. Los autores presentan los resultados y problemáticas asociadas a fogones experimentales llevados a cabo en diferentes tipos de suelos y en diferentes entornos.

En la Argentina, Pérez de Micou (1991) realizó una indagación etnoarqueológica en la Provincia de Chubut con el fin de poder discernir sobre la funcionalidad de las estructuras de combustión. García (1993) indagó sobre la relación entre las temperaturas alcanzadas por distintos combustibles y García y Zárate (1999) realizaron fogones experimentales en un sector precordillerano de la Provincia de Mendoza, para evaluar la relación entre conservación de fogones y ubicación de los mismos en diferentes estructuras geomorfológicas. Los autores concluyen que en ambientes a cielo abierto o de reparos rocosos leves, la perdurabilidad de los fogones en superficie es muy efímera, siendo el mejor indicador de la ubicación original del fogón la identificación de sustrato termo-alterado. Por otra parte, Andreoni et al. (2009) estudiaron el efecto de la carbonización en 16 especies leñosas de la Provincia de Mendoza, concluyendo que algunos taxones pueden considerarse buenos indicadores arqueológicos de altas temperaturas de combustión, mientras que otros no presentaron valor diagnóstico. Además, en un trabajo previo (Aguirre y Rodríguez 2010) hemos presentado de manera preliminar los resultados que en esta oportunidad damos a conocer detalladamente y luego de una revisión.

Una vez que el proceso de combustión ha ocurrido, se generan carbones que pueden incorporarse a los contextos arqueológicos y ser afectados por los mismos procesos post-depositacionales que afectan a los restantes vestigios arqueológicos. Desde el punto de vista tafonómico, los agentes que inciden sobre los mismos son: el viento, que puede actuar una vez finalizada la combustión o durante la misma, transportando el material fuera de su contexto original; el agua, en relación con la migración de los carbones por 
el efecto de lavado y arrastre que produce la lluvia; la temperatura, ya que los cambios y la amplitud térmica diaria afectan al material provocando fragmentación espontánea. En cuanto a los agentes bióticos, a través de la acción de animales -insectos- y de plantas -raíces-, los mismos pueden provocar tanto migraciones como fracturas del material (Scott et al. 2000, Marconetto 2008). Además de los agentes mencionados, es importante agregar aquellas prácticas sociales que intervienen en la conformación de los conjuntos de carbón arqueológico. Así, las acciones asociadas al mantenimiento y limpieza de áreas de combustión ocasionan en los carbones nuevas fragmentaciones, superficies redondeadas como también acumulaciones secundarias de combustión (Piqué y Huerta 1999). Otro factor que puede influir en la formación de los conjuntos de carbones es la presencia de productos resinosos tal como ocurre en varias especies de los géneros Fabiana (Barboza y Hunziker 1993) y Baccharis (Cazes Camarero 2003).

\section{Materiales y métodos}

Se mencionó anteriormente que los estudios antracológicos realizados en el área de estudio tienen una importante tradición. De los mismos se desprendieron diferentes preguntas de trabajo que requirieron, de manera preliminar, un abordaje de tipo experimental. Los ensayos realizados hasta el momento, a pesar de no ser numerosos, constituyen una base a partir de la cual profundizar y plantear a futuro un programa de experimentación para el área.

\section{Experimentación}

Las actividades de experimentación se llevaron a cabo en el área de estudio. Previamente, se establecieron las siguientes variables constantes: altitud sobre el nivel del mar, taxones a quemar y peso de los mismos. En cuanto a las variables no constantes (aquellas no controlables), se tomaron en cuenta los factores climáticos: temperatura ambiente al momento de encender fuego y circulación de viento. Otro factor contemplado fue el momento de dar por iniciado y terminado el experimento; en este sentido, la decisión fue dejar que el fuego se apagara naturalmente. Dado que el objetivo general de este trabajo es determinar el modo en que cada especie responde ante el fuego, consideramos adecuado no intervenir en el mantenimiento y apagado del mismo. Las quemas se realizaron en un ambiente oxidante, a cielo abierto, al reparo de un bloque mediado de roca ignimbrítica. Las acciones experimentales incluyeron distintas etapas:

1-Antecedentes bibliográficos. Realizamos una revisión de los antecedentes arqueológicos para el área a fin de reconocer las especies identificadas en el registro antracológico de diferentes sitios estudiados (Rodríguez 1998, 2000, 2004; Aguirre 2007, 2009). Ver en resultados en Tabla 2

2-Trabajo etnobotánico. Consideramos la información etnobotánica disponible para poder reconocer las especies empleadas como recursos leñosos actualmente en la localidad. Esta información proviene de distintos trabajos etnobotánicos llevados a cabo en el área de estudio (Cuello 2006; Olivera 2006; Aguirre 2009, 2012).

3-Recolección de materias primas. Las especies utilizadas en las experimentaciones proceden de los denominados Sectores Intermedios y dentro de éstos, de sectores ambientales y topográficos concretos, de acuerdo con la información etnobotánica obtenida. En estos 
sectores pudimos recolectar ocho especies leñosas propias de la flora de Antofagasta de la Sierra. Los tallos de "Tola" - Parastrephia lucida (Meyen) Cabrera - se obtuvieron en la vega del río Las Pitas y los de "Rica-Rica" -Acantholippia deserticola (Phil.) Moldenke - provienen de una de las terrazas de dicho río. Del sector de la pampa de las peñas proceden: "Tramontana" -Ephedra breana Phil.-, "Lejía" -Baccharis tola Phil -, "Cachiyuyo"-Atriplex imbricata (Moq.) D. Dietr.-, Añahua -Adesmia horrida Gillies - "Tolilla" -Fabiana punensis S. C. Arroyo - y “Leña de Lagarto" -Fabiana bryoides Phil.

4- Elaboración de los fogones. Llevamos a cabo esta tarea a una altitud de $3.600 \mathrm{msm}$, durante la estación de verano, siendo la temperatura ambiente de $20^{\circ} \mathrm{C}-25^{\circ} \mathrm{C}$ a la sombra. En el área la presión atmosférica es de una media anual de $654 \mathrm{~mm}$. Confeccionamos dos tipos de fogones, uno en piso y otro en cubeta. El fogón en piso se delimitó a partir de la limpieza del suelo a través de la eliminación de clastos de distintos tamaños que cubren el suelo desnudo de vegetación, típico de estos sectores de la Puna mientras que el fogón en cubeta consistió en la realización de una hilera de piedras y en la modificación del suelo a fin de crear una superficie cóncava (Figura 2). La estructura así generada tuvo un altura de $10 \mathrm{~cm}$ y $30 \mathrm{~cm}$ de diámetro. Es importante aclarar que optamos por trabajar con estos tipos de fogones ya que corresponden a los dos tipos de estructuras identificadas en sitios arqueológicos del área. Por otra parte, esta tipología también corresponde a la identificada en otras regiones del mundo (March y Wünsch 2003, Vallverdú et al. 2012). Durante trabajos de campo hemos observado que los pobladores actuales del área emplean principalmente estructuras similares a los denominados fogones en cubeta.

Cada especie recolectada fue pesada (Tabla 1) y en ambos tipos de fogones se quemó el mismo peso de leña $(1947,63 \mathrm{~g})$. Las ocho especies seleccionadas se quemaron conjuntamente en cada uno de los fogones, es decir que las leñas se mezclaron y se dio inicio a la ex-

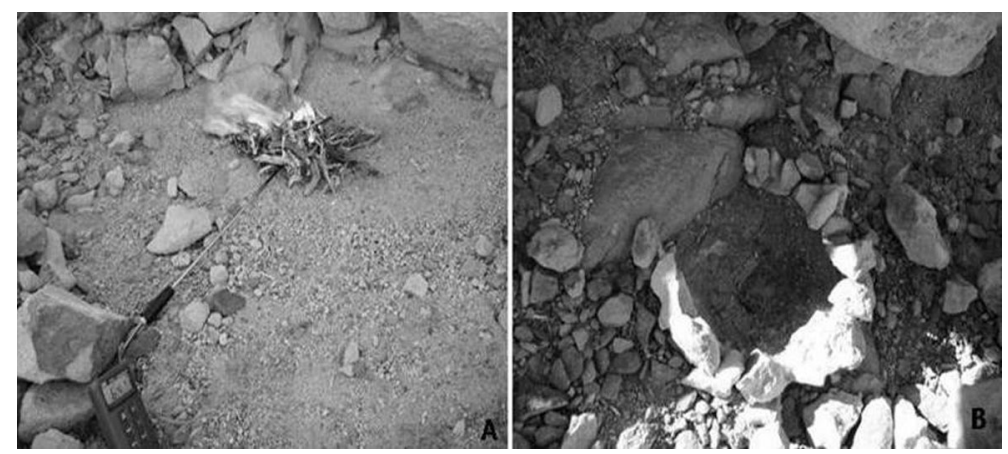

Figura 2 Fogones experimentales: A-fogón en piso B-fogón en cubeta

perimentación. Al tratarse de especies arbustivas, los tallos empleados variaron en grosor, entre los 3 y $5 \mathrm{~cm}$. Se consideró como tiempo de duración de los mismos el comienzo de las llamas y como finalización la desaparición de las mismas (brasas). Se tomó la temperatura a cada minuto, teniendo en cuenta también las variaciones de la intensidad del viento durante los experimentos. Una vez que los ensayos terminaron, se levantaron los residuos generados (ceniza y carbones). La temperatura de los fuegos fue medida con termocupla (LUFTMAN Mod. 1500 HT) en el centro de los mismos. 


\begin{tabular}{|c|c|}
\hline $\begin{array}{c}\text { Taxones quemados en } \\
\text { fogones }\end{array}$ & Peso inicial (g) \\
\hline Acantholippia deserticola & 309.45 \\
\hline Adesmia horrida & 254 \\
\hline Atriplex imbricata & 193.15 \\
\hline Baccharis tola & 259.97 \\
\hline Ephedra breana & 162.63 \\
\hline Fabiana bruoides & 272.45 \\
\hline Fabiana punensis & 303.85 \\
\hline Parastrephia lucida & 192.13 \\
\hline
\end{tabular}

Tabla 1 Especies quemadas en los fogones. Se indica el peso inicial de cada muestra

Además de las especies antes mencionadas, incorporamos a los fogones tallos de Chenopodium quinoa Willd. "Quínoa" los cuales no dejaron macrorrestos luego de la combustión (Aguirre y Rodríguez 2010). En esta versión ampliada de los ensayos que realizamos, preferimos dejar de lado los resultados obtenidos para Quínoa por no tratarse concretamente de una especie leñosa.

5-Análisis de los materiales en laboratorio. Los carbones obtenidos en el campo se describieron anatómicamente y se cuantificaron los individuos por especie. Cada carbón se fracturó según los planos: transversal, en el que se distinguen caracteres fundamentales tales como las traqueidas, elementos de vaso y anillos anuales de crecimiento; longitudinal tangencial y longitudinal radial, donde se observan las traqueidas longitudinalmente, las placas de perforación y otros elementos de la arquitectura interna de la madera, tales como traqueidas, fibras y radios (Pique y Huerta, 1991). Los fragmentos se analizaron utilizando microscopio óptico adaptado con iluminación incidente a 10, 40 y 100 X. Se tomaron fotografías con Microscopio Electrónico de Barrido de los servicios de microscopia del Museo Argentino de Ciencias Naturales Bernardino Rivadavia y de LAMENOA (CONICET-UNT).

Si bien cada taxón estaba identificado antes de llevar a cabo los ensayos de quema, al ocurrir el proceso de combustión la apariencia externa de los mismos se modificó por lo que fue necesario realizar la identificación taxonómica de cada resto de carbón. Dicha identificación se efectuó por comparación anatómica a partir de la colección y la xiloteca de referencia elaboradas a partir del material actual coleccionado en el área de estudio e identificado taxonómicamente en el Instituto de Botánica Darwinion, en cuyo herbario (SI) se encuentran depositados. Se consultó además, material actual depositado en el herbario de la Fundación Miguel Lillo (LIL). Asimismo, disponemos de fichas anatómicas para cada especie con las fotomicrografías de los cortes histológicos en distintas direcciones -transversal, longitudinal tangencial y longitudinal radial- (Aguirre 2012). También se tuvo en cuenta la bibliografía específica acerca del tema (Rodríguez 1998, 2000, 2004, Aguirre 2007).

El análisis anatómico se realizó teniendo en cuenta los caracteres que se mencionan a continuación: vasos (forma, tamaño, cantidad y disposición: porosidad), parénquima (tipo y disposición), radios (longitud y cantidad, tipo de células que los constituyen), fibras (cantidad y características), anillos de crecimiento (demarcados o no demarcados), presencia de contenidos celulares (taninos y cristales). 


\section{Material actual examinado}

Acantholippia deserticola: ARGENTINA, Catamarca, Depto. Antofagasta de la Sierra, $3600 \mathrm{~m}$ s. m., XII- 1998, Rodríguez 1 (SI)

Adesmia horrida: ARGENTINA. Catamarca. Depto. Antofagasta de la Sierra, Antofagasta de la Sierra, 3600 m s.m., III-1996 (fl), (SI) 28288; Antofagasta de la Sierra, 4000 m s.m., XII-1998 (fl), Rodríguez 13 (SI); Antofagasta de la Sierra, 3600 m s.m., XI 1999 (fl), Rodríguez 20 (SI) Atriplex imbricata.: ARGENTINA. Catamarca. Depto. Antofagasta de la Sie $\neg$ rra, Antofagasta de la Sierra, 3700 m s.m., I-1994, (SI) 28.212.

Bacharis incarum: ARGENTINA. Catamarca. Depto. Antofagasta de la Sierra, Anto $\neg$ fagasta de la Sierra, 3700 m s.m., XII-1998 (fl), Rodríguez 18 (SI)

Ephedra breana: ARGENTINA. Catamarca. Depto. Antofagasta de la Sierra, Antofangasta de la Sierra, 3700 m s.m., Cuello 147 (LIL).

Fabiana bryoides: ARGENTINA. Catamarca. Depto. Antofagasta de la Sierra, Antofargasta de la Sierra, 3650 m s.m., I-1994, SI 28217, SI 28331, (SI) 28217.

Fabiana punensis: ARGENTINA. Catamarca. Depto. Antofagasta de la Sierra, Antofagasta de la Sierra, 3650 m s.m., XII-1998, Rodríguez 17 (SI).

Parastrephia lucida: ARGENTINA. Catamarca. Depto. Antofagasta de la Sierra, Antofagasta de la Sierra, 3650 m s.m., XII-1998, Rodríguez 11 (SI).

\section{Resultados}

Del peso total inicial de leña incorporado en cada fogón, se recuperó un peso final de 116, 55 g (ceniza: 109, 64 g; carbones: 6, 91 g) en el fogón en cubeta y 69,49 g (ceniza 40,9 g; carbones: $19,59 \mathrm{~g})$ para el fogón en piso.

Además de los taxones considerados, es decir de las especies quemadas mencionadas en la Tabla 1, se obtuvieron carbones que no resistieron la aplicación de fuerza al intentar fracturarlos y en consecuencia se desintegraron impidiendo su identificación taxonómica. Para los mismos incluimos la categoría de No Identificados (32 carbones en el fogón en cubeta y 11 en el fogón en piso).

Las Figuras 3 y 4 representan las variaciones de tiempo y temperatura para ambos tipos de fogones, mientras que en la Figura 5 se puede observar, de manera comparativa, el número de fragmentos de carbón recuperados en el fogón de piso y en el de cubeta. El fogón en cubeta tuvo una duración de 52 min y el fogón en piso 50 min.

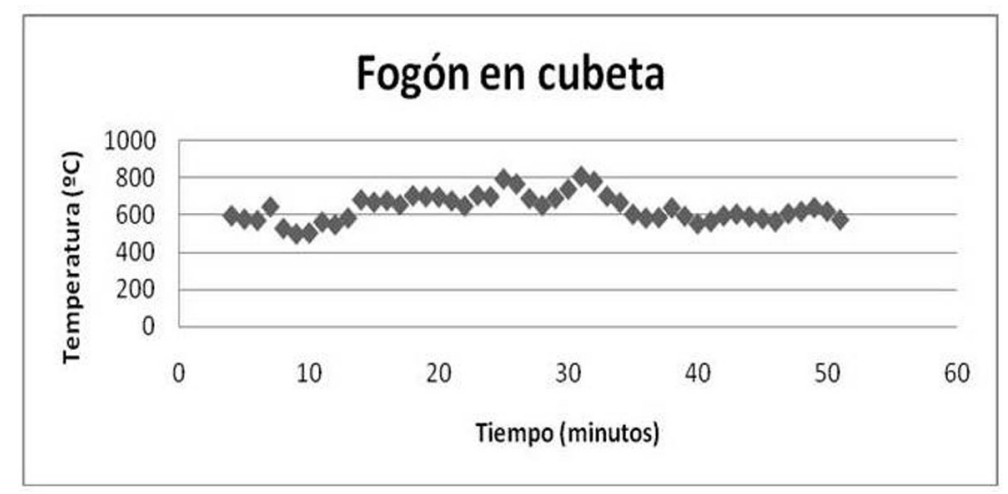

Figura 3 Valores de temperatura y tiempo para el fogón en cubeta 


\section{Discusión}

La importancia de los trabajos experimentales reside en permitir examinar supuestos arqueológicos y contribuir así a la construcción de marcos de referencia acerca del comportamiento humano en el pasado (Ascher 1961, Coles 1979, Nami 1991).

\begin{tabular}{|c|c|c|c|}
\hline $\begin{array}{l}\text { Taxón/Nombre } \\
\text { vernacular }\end{array}$ & Usos actuales en ANS* & $\begin{array}{c}\text { Registro } \\
\text { Arqueológico }\end{array}$ & Referencia \\
\hline Calycera sp. (Cuerno) & Leña $^{4}$ & No registrada & 4 \\
\hline Mulinun sp. (Espinillo) & Leña $^{4}$ & No registrada & 4 \\
\hline $\begin{array}{c}\text { Acantholippia deserticola } \\
\text { (Rica-Rica) }\end{array}$ & $\begin{array}{l}\text { Leña }^{1}, \text { medicinal }^{3} \text {, } \\
\text { tintórea }^{3}\end{array}$ & Carbón²,7 & $1,2,3,7$ \\
\hline $\begin{array}{c}\text { Acantholippia salsoloides } \\
\text { (Rica-Rica) }\end{array}$ & Medicinal $^{4}$, Leña ${ }^{4}$ & No registrada & 4 \\
\hline $\begin{array}{c}\text { Adesmia caespitosa } \\
\text { (Cuerno) }\end{array}$ & Leña $^{4}$ & No registrada & 4 \\
\hline $\begin{array}{l}\text { Adesmia erinacea (Añahua } \\
\text { blanca) }\end{array}$ & Leña $^{4}$ & No registrada & 4 \\
\hline Adesmia horrida (Añahua) & Leña $^{1}$ & Carbón'2,5,6,7 & $1,2,5,6,7$ \\
\hline $\begin{array}{c}\text { Adesmia subterranea } \\
\text { (Cuerno) }\end{array}$ & Leña $^{1}$ & Carbón ${ }^{2}$ & 1,2 \\
\hline $\begin{array}{l}\text { Atriplex imbricata } \\
\text { (Cachivuvo) }\end{array}$ & Leña $^{1}$ & Carbón ${ }^{2}$ & 1,2 \\
\hline Baccharis tola (Lejía) & Leña $^{1,4}$, Comestible $^{3}$ & Carbón'2,5,6,7 & $\begin{array}{c}1,2,3,4,5 \\
6.7\end{array}$ \\
\hline $\begin{array}{l}\text { Chuquiraga atacamensis } \\
\text { (Monte de Suri) }\end{array}$ & Medicinal $^{3}$ & Carbón 2,5 & 2,3 \\
\hline $\begin{array}{l}\text { Ephedra breana } \\
\text { (Tramontana) }\end{array}$ & $\begin{array}{l}\text { Leña }{ }^{1}, \text { medicinal }^{3} \text {, } \\
\text { tintórea }^{3}\end{array}$ & Carbón ${ }^{2}$ & $1,2,3$ \\
\hline $\begin{array}{c}\text { Fabiana bryoides (Leña de } \\
\text { Lagarto) }\end{array}$ & $\begin{array}{c}\text { Leña }^{1,4}, \text { medicinal }^{3}, \\
\text { sahumar }^{3}\end{array}$ & Carbón' $2,5,6,7$ & $\begin{array}{c}1,2,3,4,5 \\
6,7\end{array}$ \\
\hline Fabiana punensis (Tolilla) & Leña ${ }^{1,4}$ & Carbónn²,5,6,7 & $\begin{array}{c}1,2,4,5,6, \\
7\end{array}$ \\
\hline $\begin{array}{c}\text { Junellia seriphioides (Leña } \\
\text { de Lagarto) }\end{array}$ & Leña $^{4}$ & No registrada & 4 \\
\hline $\begin{array}{c}\text { Neosparton ephedroides } \\
\text { (Badre) }\end{array}$ & Leña ${ }^{1,4}$ & No registrada & 1,4 \\
\hline $\begin{array}{c}\text { Neuontobotrys tarapacana } \\
\text { (Chuchar) }\end{array}$ & Leña $^{1}$ & Carbón ${ }^{2}$ & 1,2 \\
\hline Parastrephia lucida (Tola) & Leña $^{1}$, medicinal ${ }^{3}$ & Carbón' $2,5,6,7$ & $1,2,5,6,7$ \\
\hline $\begin{array}{c}\text { Parastrephia } \\
\text { quadrangularis (Chacha) }\end{array}$ & $\begin{array}{c}\text { Leña }{ }^{1}, \text { medicinal, } \\
\text { sahumar }^{3}\end{array}$ & Carbón' $2,5,6,7$ & $\begin{array}{c}1,2,3,5,6 \\
7\end{array}$ \\
\hline $\begin{array}{c}\text { Parastrephia } \\
\text { phulacaeformis (Tola) }\end{array}$ & Leña $^{4}$ & No registrada & 4 \\
\hline $\begin{array}{l}\text { Senecio santelicis } \\
\text { (Chachacoma) }\end{array}$ & No registra & Carbón ${ }^{2,5,6}$ & $2,5,6$ \\
\hline $\begin{array}{l}\text { Senecio subulatus } \\
\text { (Mocoraca) }\end{array}$ & Leña $^{4}$ & No registrada & 4 \\
\hline
\end{tabular}

Tabla 2. *Abreviatura de Antofagasta de la Sierra. Referencias: 1 Aguirre 2009, 2 2012; 3Cuello 2006; 4Olivera 2006; 5Rodríguez 1996-1997 


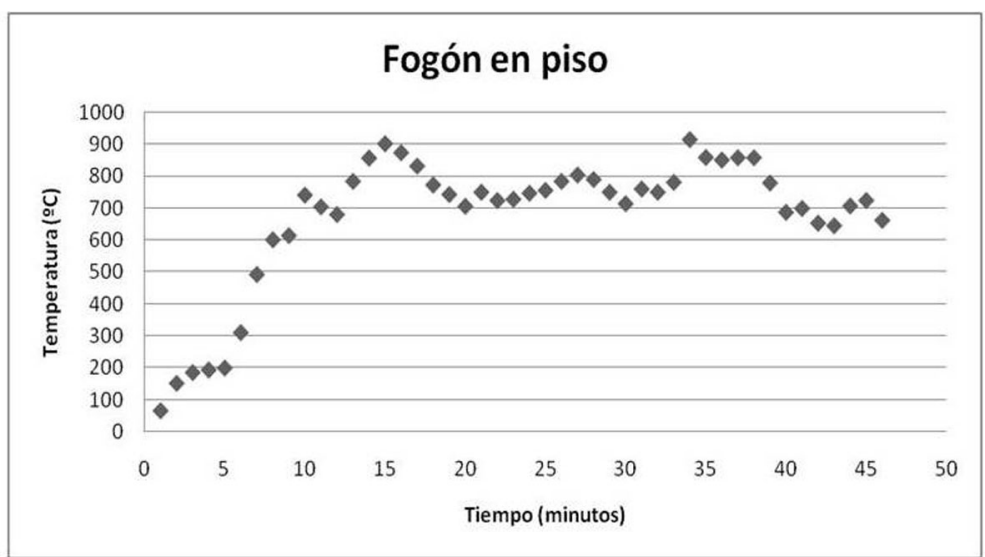

Figura 4 Valores de temperatura y tiempo para el fogón en piso

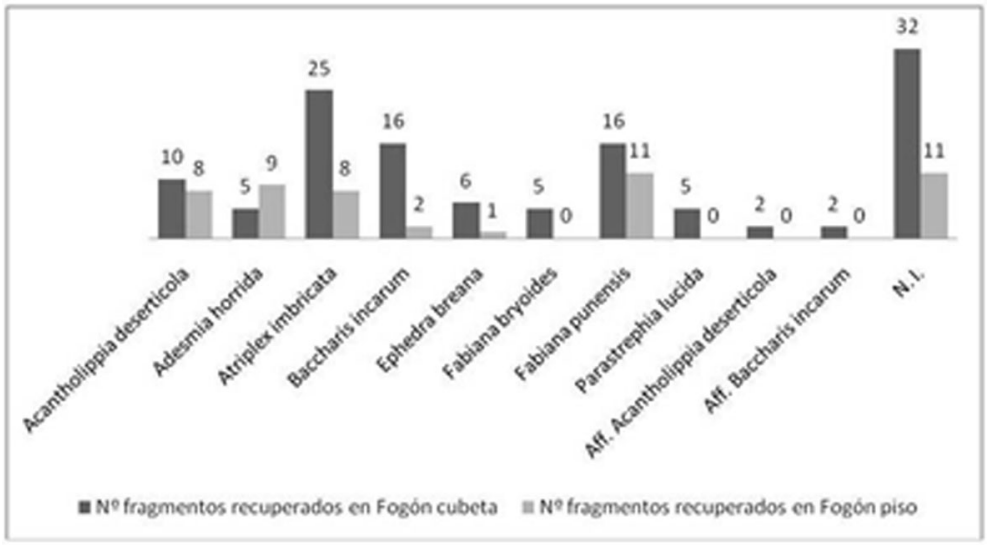

Figura 5. Número de carbones recuperados en fogones en cubeta y en piso.

Los objetivos planteados en este trabajo se abordaron desde una perspectiva experimental y los resultados obtenidos contribuyeron a elaborar una serie de observaciones que pueden ayudar a interpretar futuras investigaciones antracológicas en el área.

Las quemas realizadas en dos tipos distintos de fogones arrojaron diferencias en cuanto a la representación de las especies leñosas empleadas luego de ocurrido el proceso de combustión. De las ocho especies consideradas, solo seis fueron identificadas en el fogón en piso, mientras que en el fogón en cubeta se recuperaron restos de carbón de todas las especies quemadas.

Por otra parte, el número de restos de carbón generados correspondientes a cada especie, en cada tipo de fogón, fue también variable. En este sentido, el mayor número de fragmentos de carbón recuperados en el fogón en cubeta corresponden a la especie Atriplex imbricata (Cachiyuyo), de la cual se utiliza el tallo como leña en el norte de Chile, por lo general cuando no hay otras plantas disponibles (Villagrán y Castro 2004). Pérez (2006) señala su empleo actual como especie forrajera en la localidad de El Peñón (Dpto. Antofagasta de la Sierra).

Las especies Baccharis tola (Lejía) y Fabiana punensis (Tolilla) aportaron cada una 16 fragmentos de carbón para el fogón en cubeta. En la actualidad, Lejía es catalogada como un buen combustible por los pobladores del área (Aguirre 2009) mientras que Tolilla es empleada también como combustible, con fines tintóreos y veterinarios en la localidad de El Peñón (Pérez 2006). 
En cuanto a las especies Acantholippia deserticola (Rica-Rica) y Ephedra breana (Tramontana), éstas generaron 10 y 6 fragmentos de carbón respectivamente en el fogón en cubeta, la primera es considera uno de los mejores combustibles en Antofagasta de la Sierra (Aguirre 2009). Villagrán y Castro (2004) señalan que la raíz proporciona leña principalmente cuando está seca. Asimismo, según datos etnobotánicos registrados por una de las autoras de este trabajo en el área, esta especie ha sido identificada como leña de verano (Aguirre 2009).

Por último, Adesmia horrida (Añahua), Fabiana bryoides (Leña de Lagarto) y Parastrephia lucida (Tola) aportaron la menor cantidad de fragmentos de carbón en el fogón en cubeta. Diversas especies del género Adesmia sp., son consideradas por comunidades actuales como muy buenos combustibles (Villagrán y Castro 2004). Así por ejemplo, en un estudio de carácter etnobotánico realizado en el área registramos el taxón A. horrida como leña de invierno (Aguirre 2009). En cuanto a Fabiana bryoides, en la localidad de El Peñón se utiliza para sahumar el ganado y las casas para la celebración del $1^{\circ}$ de Agosto y para espantar los males; tiene también utilidad como combustible (Rodríguez 1998, 2000; Pérez 2006). Villagrán y Castro (2004) dan cuenta también de su uso como leña en el Salar de Atacama, en el norte de Chile. Además, en Susques (Puna jujeña) Fabiana sp. es usada como leña pero no es considerada buen combustible (Joly et al. 2009). En relación con Parastrephia lucida (Tola), la misma corresponde a un arbusto resinoso densamente hojoso que crece próximo a los cursos de agua y es abundante en el Tolar (Villagrán y Castro 2004, Cuello 2006). En general, las Tolas son catalogadas como el mejor combustible tanto en Susques (Puna de Jujuy) (Joly et al. 2009) como en Antofagasta de la Sierra (Aguirre 2009).

En cuanto al número de fragmentos de carbón preservados en el fogón en piso, la situación es diferente a la registrada para el fogón en cubeta. En primer lugar, la especie mejor representada es Fabiana punensis (Tolilla), seguida por Adesmia horrida (Añahua), Acantholippia deserticola (Rica-Rica) y Atriplex imbricata (Cachiyuyo), mientras que Baccharis tola (Lejía) y Ephedra breana (Tramontana) solo aportaron 2 y 1 fragmento de carbón respectivamente. La mayor diferencia registrada entre ambos fogones tiene que ver con la ausencia de restos de las especies Parastrephia lucida (Tola) y Fabiana bryoides (Leña de Lagarto) en el fogón en piso.

Los datos etnobotánicos relevados para el área señalan a Parastrephia lucida (Tola), Ephedra breana (Tramontana) y Baccharis tola (Lejía) como los tres mejores combustibles, siendo además las dos primeras especies empleadas durante el invierno (Aguirre 2009). $\mathrm{Al}$ integrar estos datos con los registrados luego de los ensayos experimentales, se observa que Tola no generó restos de carbón para el fogón en piso mientras que en el fogón en cubeta está representada por solo 5 fragmentos. En el caso de Tramontana y Lejía, ambos taxones se recuperaron en los fogones diseñados pero la mejor representada es el segundo.

Consideramos que las diferencias observadas en cuanto a la representación de estos combustibles en ambos tipos de fogones tienen que ver en primer lugar con la arquitectura de los mismos; las características morfológicas del fogón en cubeta habrían impedido la acción directa del viento durante la combustión. En el ambiente puneño el viento es constante y fue posible observar mientras se desarrollaban los ensayos, el arrastre de carbones y cenizas ocasionado por el viento, principalmente en el fogón en piso. Por otra parte, la forma del fogón en cubeta habría permitido que las temperaturas alcanzadas fueran más estables que las del fogón en piso, al tiempo que se recuperó también un mayor peso de cenizas. 
Las conclusiones a las que hemos arribado son coincidentes con las descritas por March (1992) en relación con la estabilidad de las temperaturas de los fogones en cubeta y al mayor número de fragmentos de carbón en este tipo de estructuras.

Además de la morfología del fogón en cubeta, la mejor representación de Baccharis incarum puede relacionarse con el peso quemado de esta leña. Hemos recolectado 259,97 g de leña de B. incarum mientras que para Ephedra breana y Parastrephia lucida agregamos a cada fogón un peso total de 162,63 g y 192,13g respectivamente.

Es posible comparar además los resultados experimentales obtenidos con los datos disponibles para diferentes sitios del área. En el sitio Quebrada Seca 3 los combustibles leñosos mejor representados corresponden en primer lugar a Adesmia horrida, seguida por $P$. quadrangularis, P. lucida, Baccharis tola, Fabiana bryoides, Senecio santelicis, Fabiana punensis y Sysimbrium phillipianum (Rodríguez 2000), mientras que para el sitio Punta de la Peña 9 son Adesmia horrida, Acantholippia deserticola, Fabiana bryoides y P. quadrangularis (Rodríguez 2004). Por otra parte, en Peñas Chicas 1.3, Adesmia sp., Acantholippia sp., Parastrephia sp. y Fabiana sp. son los taxones más frecuentes (Aguirre 2007). Los datos generados para el sector de la Quebrada Miriguaca indican para el sitio Alero Sin Cabeza, ejemplares de los géneros Adesmia sp., Acantholippia sp., Fabiana sp., Parastrephia sp., Aff. Parastrephia sp., Aff. Adesmia sp., Aff. Fabiana sp. y especímenes de Acantholippia deserticola, Adesmia horrida, Atriplex imbricata, Chuquiraga atacamensis Fabiana bryoides, Fabiana punensis, Parastrephia lucida, Parastrephia quadrangularis y Neuontobotrys tarapacana (Aguirre 2012).

Pudimos comprobar que en los casos arqueológicos los taxones Adesmia horrida y Acantholippia deserticola son los mejores posicionados. Durante los ensayos experimentales llevados a cabo, quemamos un peso de 309,45 g de Acantholippia deserticola y $254 \mathrm{~g}$ de Adesmia horrida, recuperando carbones de ambas especies en los dos tipos de fogones realizados. Sin embargo, en ningún caso son estas especies las que aportaron un mayor número de fragmentos de carbón en los ensayos experimentales. Pero por otra parte, Acantholippia deserticola y Adesmia horrida están mejor representadas experimentalmente que las especies consideradas como los mejores combustibles (Parastrephia lucida, Ephedra breana y Fabiana bryoides) para el área. Esto nos lleva a pensar que durante el pasado se habría hecho uso de considerables volúmenes de leña, disponible en el área circundante a los sitios de habitación, las cuales habrían sido complementadas con otras especies que tienen mejores propiedades combustibles.

Nuestras observaciones se ven reforzadas además con los datos generados por Joly (2008) en la Puna de Jujuy, donde experimentó con la quema de Adesmia sp. cf "gruesa", Fabiana densa y Parastrephia lepidophylla. Los resultados obtenidos señalan que la primera especie alcanzó una temperatura mayor a los $300^{\circ} \mathrm{C}$ con una duración de 5 horas de combustión mientras que la tercera logró una temperatura mayor a $400^{\circ} \mathrm{C}$ pero una duración de combustión mucho menor $(2 \mathrm{~h} 10 \mathrm{~m})$. Entonces, si bien la Tola (P. lepidophylla), tanto en la Puna de Jujuy como de Catamarca, es considerada como el mejor combustible, su respuesta ante el fuego es diferente a la de Adesmia sp. Esta última genera fuegos más duraderos que los de Parastrephia sp., lo cual es necesario para actividades domésticas tales como la cocción de alimentos, iluminación o calor.

Por otra parte, los resultados preliminares que hemos obtenido plantean la importancia de definir previamente cómo llevar a cabo durante las tareas de excavación y de laboratorio, la recuperación y el muestreo de los carbones arqueológicos. En un sitio los carbones 
pueden presentarse bajo dos formas principales: dispersos en los sedimentos arqueológicos o concentrados (Chabal 1992). Estas dos formas de presentación definirán las técnicas de recuperación y muestreo a emplear, repercutiendo posteriormente en nuestras interpretaciones sobre el caso de estudio que se considere. Así, en la indagación experimental que hemos llevado a cabo, la cual corresponde a carbones concentrados, observamos la importancia de la recuperación del total de fragmentos preservados hasta el momento de la excavación y del análisis. Además, si es factible se consideran todos los fragmentos, ya que tal como señala Badal García (1992) la unidad de observación en antracología es el fragmento de carbón. No obstante, reconocemos que en algunos casos el estudio del total de los fragmentos no es viable por motivos técnicos, económicos o de tiempo. En estos casos se requiere plantear un muestreo en laboratorio que permita generar una visualización de las especies preservadas hasta la intervención del arqueólogo. Entonces ya no hablamos de todas las especies empleadas durante el funcionamiento de la estructura de combustión ya que nuestros resultados experimentales señalan la posibilidad de que ciertos taxones introducidos a la combustión no estén representados luego en el conjunto de macrorrestos.

\section{Conclusiones}

En este trabajo evaluamos la reacción de ocho especies leñosas combustibles ante la acción del fuego. Asimismo, integramos los resultados experimentales obtenidos con los generados en investigaciones etnobotánicas y antracológicas realizadas en el área de estudio. Para llevar a cabo la primer tarea durante el proceso de combustión, diseñamos dos tipos de fogones, uno en cubeta y otro en piso. Observamos que las tres especies leñosas (Parastrephia lucida, Ephedra breana y Fabiana bryoides) consideradas para el área como los mejores combustibles, tienen tanto a nivel arqueológico como experimental, una mediana a baja representación de fragmentos de carbón.

A partir de lo dicho en el párrafo anterior, concluimos que ciertas especies incorporadas al proceso de combustión no generan residuos macroscópicos, lo cual se debería principalmente al tipo de estructura de combustión que se emplee y a las características climáticas predominantes durante las quemas. Además de estos dos elementos, deben tenerse en cuenta las características físicas y químicas de las maderas quemadas y los procesos post-depositacionales. Por este motivo, la ausencia de restos carbonizados de una determinada planta no implica que la misma no haya sido utilizada efectivamente. Es en estos casos donde los datos etnobotánicos sobre el uso de los recursos vegetales constituyen una vía importante para evaluar criterios de selección, frecuencia y estacionalidad de los combustibles leñosos. Por otra parte, esta representación diferencial de las especies leñosas debe tenerse en cuenta al momento de plantear las técnicas de recuperación y el diseño de muestro de carbón que se utilizará en el estudio arqueológico.

En cuanto a las temperaturas alcanzadas por las especies locales quemadas, los valores registrados amplían las expectativas acerca de las posibles actividades de producción tecnológica que podrían haberse llevado a cabo en la región. En nuestro caso podemos decir que el área de Antofagasta de la Sierra cuenta con recursos leñosos que pueden alcanzar altas temperaturas.

A partir de los resultados presentados, consideramos que se abre la necesidad de desarrollar un programa de experimentación más amplio para el área a fin de continuar indagando sobre la reacción de las especies leñosas ante el fuego y, además, entrecruzar este tipo de diseños experimentales de fogones con investigaciones referidas a actividades tales como la producción de cerámica y metales. 
Agradecimientos: Esta investigación contó con el apoyo de los proyectos CIUNT 26/G404 dirigido por el Lic. Carlos Aschero y PICT 2006 No 2264 dirigido por la Dra. Pilar Babot. Agradecemos además los comentarios y sugerencias de los evaluadores que sin duda contribuyeron a mejorar este trabajo.

\section{Bibliografía citada}

Aguirre, M. G.

2007 Arqueobotánica del sitio Peñas Chicas 1.3 (Antofagasta de la Sierra, Catamarca, Argentina). Paleoetnobotánica del cono sur: estudios de casos y propuestas metodológicas, (ed. por B. Marconetto, M. P. Babot y N. Oliszewski), pp. 179-195. Ferreyra Editores, Córdoba.

2009 Conocimiento y uso de los recursos leñosos en la Puna meridional argentina. Actas de las III Jornadas de Jóvenes Investigadores U N T. Versión CD-ROM (sin paginación).

2012 Recursos vegetales: uso, consumo y producción en la Puna meridional argentina (5000-1500 AP). Tesis Doctoral. Facultad de Ciencias Naturales y Museo. Univ. Nacional de La Plata, La Plata.

Aguirre, M. G. y M. F. Rodríguez

2010 La experimentación como forma de aproximación a la tafonomía del registro antracológico. Actas del XVII Congreso Nacional de Arqueología Argentina, pp. 1187-1191. Universidad Nacional de Cuyo.

Allué, E., I. Euba, I. Cáceres, M. Esteban y M. J. Pérez

2005 Experimentación sobre recogida de leña en el parque faunístico de los pirineos "Lacuniacha" (Huesca). Una aproximación a la tafonomía del registro antracológico. Actas del VI Congreso Ibérico de Arqueometría. Avances en Arqueometría, pp. 295-303. Universitat de Girona.

\section{Andreoni, D., A. Gil y A. Capparelli}

2010. Efectos de la carbonización en especies leñosas de las provincias fitogeográficas patagónica y del monte (Mendoza, Argentina): una perspectiva arqueológica. Tradiciones y transformaciones en etnobotánica, (ed. por M. L. Pochettino, A. Ladio y P. Arenas), pp. 33-37. CYTED-Programa Iberoamericano Ciencia y Tecnología para el desarrollo, Jujuy.

Ascher, R.

1961 Experimental Archaeology. American Anthropologist 63 (4): 793-816.

Badal García, E.

1992 L'anthracologie préhistorique: a propos de certains problèmes méthodologiques. Les Charbons de bois les anciens Écosystèmes et le Rôle de L'Homme. Bulletin de la Société Botanique de France Actualités Botanique 139: 167-189.

Badal, E., Y. Carrión, D. Rivera y P. Uzquiano

2003 La arqueobotánica en cuevas y abrigos: objetivos y métodos de muestreo. La recogida de muestras en arqueobotánica: objetivos y propuestas metodológicas. La gestión de los recursos vegetales y la transformación del paleopaisaje en el Mediterráneo occidental, pp. 19-29. Encuentro del Grupo de Trabajo de Arqueobotánica de la Península Ibérica. Bellaterra, Barcelona.

Barboza, G. y A. Hunziker

1993 Estudios de Solanaceae XXXIV. Revisión taxonómica de Fabiana. Kurtziana 22: 109-153. 
Behrensmeyer, A. K. y S. M. Kidwell

1985 Taphonomy's Contributions to Paleobiology. Paleobiology 11: 105-119

Braadbaart, F. y I. Poole

2008 Morphological, chemical and physical changes during charcoalification of wood and its relevance to archaeological contexts. Journal of Archaeological Science 35: 2434-2445.

Braadbaart, F., I. Poole y A. A. Van Brussel

2009 Preservation potential of charcoal in alkaline environments: an experimental approach and implications for the archaeological record. Journal of Archaeological Science 36 (8): 1672-1679.

Bueno Zárate, J.

1987. Las maderas como combustible. Revista Forestal del Perú 14(2): 1-9.

Byrne, C. E. y D. C. Nagle.

1997 Carbonization of wood for advanced materials applications. Carbon 35(2): 259-266.

Cabrera, A. L.

1976 Regiones Fitogeográficas Argentinas. Enciclopedia Argentina de Agricultura y Jardinería. (ed. por W. F. Kugler), pp. 1-85. Editorial Acme, Buenos Aires.

Cabrera, A. L. y A. Willink

1980 Biogeografía de América Latina. Secretaría General de la Organización de los Estados Americanos. Serie de Biología. Monografía No 13, Washington D. C.

Cazes Camarero, P.

2003 Asteraceae biodinámicas del Herbario del Museo "Juan A. Domínguez" (Facultad de Farmacia y Bioquímica - UBA). Dominguezia 19(1):5-19.

Chabal, L.

1992 La représentativité paléo - écologique des charbons de bois archaéologiques issus du bois de feu. Les Charbons de bois les anciens Écosystèmes et le Rôle de L'Homme. Bulletin de la Société Botanique de France Actualités Botanique 139: 213-236.

Coles, J.

1979 Archaeology of experiment. University of Cambridge. Cambridge.

Cuello, A. S.

2006 Guía ilustrada de la flora de Antofagasta de la Sierra - Catamarca (Puna Meridional Argentina). Curso de entrenamiento para la obtención del grado de Licenciatura en Ciencias Biológicas (orientación Botánica). Facultad de Ciencias Naturales e IML. Universidad Nacional de Tucumán, Tucumán.

Demirbas, A.

2004 Combustion characteristics of different biomass fuels. Progress in Energy and Combustion Science 30(2): 219-230.

Efremov, J. A.

1940 Taphonomy: New Branch of Paleontology. Pan-American Geologist 74: 81-93. 
Fahn, A.

1974 Anatomía vegetal. H. Blume Ediciones. Madrid.

García, L.

1993 Experimentación en Inca Cueva: arcillas, fogones y combustibles. Arqueología 3:69-91.

García, A. y M. Zarate

1999 Perdurabilidad y cambio de fogones experimentales en la precordillera mendocina. Arqueología 9: 113-130.

González Urquijo, J. E, J. Ibáñez Estévez, M. Moreno García, L. Peña Chocarro y L. Zapata Peña 2005 An ethnoarchaeological Project in the Eestern Rif (Northern Morocco): First results. Quaderni del Laboratorio di Archeobiologia 1: 21-32.

Haber, A.

1992 Pastores y pasturas. Recursos forrajeros en Antofagasta de la Sierra (Catamarca), en relación la ocupación Formativa. Shincal 2: 15-23.

Joly, D. R.

2008 Étude de la gestion du combustible osseux et végétal dans les stratégies adaptatives des chasseurs-cueilleurs et des groupes agro-pastoraux d'Argentine durant l'Holocène. Tesis Doctoral . Universidad de Rennes 1.

Joly, D., R. March, D. Marguerie y H. Yacobaccio

2009 Gestion des combustibles dans la province de Jujuy (Puna, Argentine) depuis l'Holocène ancien: Croisement des résultats ethnologiques et anthracologiques. Gestion des combustibles au Paléolithique et au mésolithique Nouveaux outils, nouvelles interprétations, (ed. por I. ThéryParisot, I., S. Costamagno y A. Henry), pp. 39-56. BAR International series, 1914, Oxford.

March, R.

1992 L 'utilisation du bois dans les foyers prehistoriques: une approche expérimentale, en Les Charbons de bois les anciens Écosystèmes et le Rôle de L'Homme. Bulletin de la Société Botanique de France 139: 245-253.

March, R. J. y G. Wünsch

2003 Loupes et lentilles obscures: A propos de la fonction des structures de combustion.

Colloque internationale de Beaune Le feu domestiques et ses structures, pp. 1-20.

March R., A. Lucquin, D. Joly, J. C. Ferreri y M. Muhieddine

2012 Processes of formation and alteration of archaeological fire structures: views on complexity based on experimental approaches. Journal of archaeological method and theory DOI 10.1007/s10816-012-9134-7 47.

Marconetto, M. B.

2008 Recursos forestales y el proceso de diferenciación social en tiempos Prehispánicos en el Valle de Ambato, Catamarca, Argentina. South American Archaeology Series 3. BAR International Series.

Martínez Carretero, E.

1995 La Puna argentina: delimitación general y división en distritos florísticos. Boletín de la Sociedad Argentina de Botánica 31(1-2): 27-40. 
McParland, L. C., M. E. Collison, A. Scott, G. Campbell y R. Veal

2010 Is vitrification in charcoal a result of high temperature burning of wood? Journal of Archaeological Science 37(10): 2679-2687.

Nami, H. G.

1991 Algunas reflexiones teóricas sobre arqueología y experimentación. Shincal 3(1): 151-168.

Nirmal Kumar, J. I., K. Patel, R. N. Kumar y R. Kumar Bhoi

2011 An evaluation of fuelwood properties of some Aravally mountain tree and shrub species of Western India. Biomass and Bioenergy 35(1): 411-414.

Olivera, D. E.

1992 Tecnología y estrategias de adaptación en el Formativo (Agro-alfarero Temprano) de la puna meridional argentina. Un caso de estudio: Antofagasta de la Sierra (Pcia. de Catamarca, R.A.). Tesis Doctoral. Facultad de Ciencias Naturales y Museo. Universidad Nacional de La Plata, La Plata.

2006 Recursos bióticos y subsistencia en sociedades agropastoriles de la Puna Meridional Argentina. Comechingonia 9: 19-55.

Pérez, E. L.

2006 Las plantas utilizadas por la comunidad de Antofagasta de la Sierra, Puna catamarqueña, Argentina. Curso de entrenamiento para la obtención del grado de Licenciatura en Ciencias Biológicas (orientación Botánica). Facultad de Ciencias Naturales e IML. Universidad Nacional de Tucumán, Tucumán.

Pérez de Micou, C.

1991 Fuegos, fogones y señales. Una aproximación etnoarqueológica a las estructuras de combustión en el Chubut medio. Arqueología 1: 125-150.

Picornell Gelabert, L.

2009 Antracología y etnoarqueología. Perspectivas para el estudio de las relaciones entre las sociedades humanas y su entorno. Complutum 20(1): 133-151.

Piqué i Huerta, R.

1999 Producción y uso de combustible vegetal arqueológico: Una evaluación arqueológica. Treballs d'Etnoarqueología 3. Universidad Autónoma de Barcelona. Consejo Superior de Investigaciones Científicas. Madrid.

Piqué i Huerta, R. y J. M. Piqué i Huerta

1991 Aplicación del tratamiento de imágenes digitalizadas al análisis antracológico: un ensayo de determinación automática. Arqueología: Nuevas Tendencias. pp 115-129. Consejo superior de investigaciones científicas. Madrid.

Prior J. y K. Alvin

1983 Structural changes on charring woods of Dichrostachys and Salix from southern Africa. IAWA Bulletin 4 (4): 197-206.

Rodríguez, M. F.

1998 Arqueobotánica de Quebrada Seca 3: Recursos vegetales utilizados por cazadores-recolectores durante el período Arcaico en la Puna Meridional Argentina. Tesis Doctoral. Facultad de Ciencias Exactas y Naturales. Universidad de Buenos Aires, Buenos Aires. 
2000 Woody plant species used during the Archaic period in the Southern Argentine Puna. Archaeobotany of Quebrada Seca 3. Journal of Archaeological Science 27 (4): 341 - 361.

2004 Woody plant resources in the Southern Argentine Puna. Punta de la Peña 9 archaeological site. Journal of Archaeological Science 31 (10): 1361 - 1372.

Scarano, E. R., H. M. Pucciarelli, M. Crivos y M. Prati

1994 Estado actual de la experimentación antropológica en Argentina. Interciencia 19(4): 191-195.

Schiffer, M. B.

1972 Archaeological context and systemic context. American Antiquity 37: 56-165.

1975 Archaeology as behavioral science. American Anthropologist. New Series 77(4): 836848.

Scott, A., J A. Cripps, M. E. Collinson y G. J. Nichols

2000 The taphonomy of charcoal following a recent heath land fire and some implications for the interpretation of fossil charcoal deposits. Palaeogeography, Palaeoclimatology and Palaeoecology 164: 1-31.

Théry-Parisot, I., L. Chabal y J. Chrzazvzez

2010 Anthracology and taphonomy, from wood gathering to charcoal analysis. A review of the taphonomic processes modifying charcoal assemblages, in archaeological contexts. Palaeogeography, Palaeoclimatology, Palaeoecology 291(1-2): 142-153.

Troll, C.

1958 Las culturas superiores andinas y el medio geográfico. Revista del Instituto de Geografía (Lima) 5: 3-55.

Vallverdú J., S Alonso, A. Bargalló, R. Bartrolí, G. Campeny, A. Carrancho, I. Expósito, M. Fontanals, J. Gabucio, B. Gómez, J. Maria Prats, P. Sañudo, À. Solé, J. Vilalta y E. Carbonell 2012 Combustion structures of archaeological level $\mathrm{O}$ and mousterian activity areas with use of fire at the Abric Romaní rockshelter (NE Iberian Peninsula). Quaternary International 247: 313-324.

Villagrán, C. y V. Castro

2004 Ciencia indígena de los Andes del Norte de Chile. Editorial Universitaria. Santiago de Chile.

Werkelin, J., D. Lindberg, D. Boström, B. J. Skrifvars y M. Hupa

2011 Ash-forming elements in four Scandinavian wood species part 3: Combustion of five spruce samples. Biomass and Energy 35(1): 725-733.

Zapata Peña, L., L. Peña Chocarro, J. J. Ibañez Estevez y J. E. González Urquijo 2003 Ethnoarchaeology in the Moroccan Jebala (Western Rif): Wood and dung as fuel. Africa Prehistorica 15: 163-175. 